Voix et Images

volxetimages

\title{
Lecture/écriture de Roland Giguère : « les Mots-flots »
}

\section{André Gervais}

Volume 3, numéro 2, décembre 1977

Victor-Lévy Beaulieu

URI : https://id.erudit.org/iderudit/200108ar

DOI : https://doi.org/10.7202/200108ar

Aller au sommaire du numéro

\section{Éditeur(s)}

Les Presses de l'Université du Québec

\section{ISSN}

0318-9201 (imprimé)

1705-933X (numérique)

Découvrir la revue

\section{Citer cet article}

Gervais, A. (1977). Lecture/écriture de Roland Giguère : « les Mots-flots ». Voix et Images, 3(2), 302-319. https://doi.org/10.7202/200108ar d'utilisation que vous pouvez consulter en ligne.

https://apropos.erudit.org/fr/usagers/politique-dutilisation/ 


\section{Lecture/écriture de Roland Giguère: «les Mots-flots»}

[...] le premier flot de notre Seine par ces mots déjà abondant et nourri prend son cours, $[. .$.

Francis Ponge 1

S'égarer, selon Giguère, serait s'égarer en l'écriture: c'est, selon Mallarmé, donner l'initiative aux mots. Risquer d'aller hors du droit chemin de la représentation et de la vérité, pour aller plus loin et, pour tout dire, en quelque part: dans le texte, dans la matérialité luisante et mate de son envers et de son avers ${ }^{2}$. Quelque aversion idéaliste qu'il y ait ici particulièrement, écrire serait être sous les armes, par lesquelles il s'agit de passer afin de les prendre, plus radicalement d'être pris par elles, d'être passé par elles, d'être fait d'armes: non un poète occupé, non un poète engagé, non un poète militant, mais un poète, fait d'armes. Écrire serait faire ses premières armes, se mettre en forme, et leur donner l'initiative: faire ses premières armes blanches et leur donner l'iniative, c'est poursuivre l'écriture noir sur blanc et blanc sur noir, c'est non combattre, mais battre.

1 Les mots-flots viennent battre la plage blanche

2 où j'écris que l'eau n'est plus l'eau

3 sans les lèvres qui la boivent

4 les mots-flots couronnant le plus désertique îlot

5 le lit où je te vois nager la nuit

6 et la paupière qui te couvre comme un drap

7 au versant abrupt du matin

8 quand tout vient se fracasser sur la vitre

Les mots-flots, titre et incipit des quatre premières strophes, matrice d'un texte où une mariée devient mère. Les flots viennent battre, heurter, se briser contre; les mots viennent faire barre, rature (l'eau n'est plus l'eau), lignes d'écriture. Les mots-flots viennent faire texte: ce texte-mer-ci. Casser des mots sur les flots, ressac des flots sur les mots. Sous la plage, la page: plage

- (barre de fraction).

page

Ça se fractionne où/ou ça s'unit:

mots-flots (trait d'union). 
Le vers, recto (flots) et l'envers, verso (mots):

flots

mots-flots

mots

A l'union, c'est le verso - à la fraction, c'est le recto - qui bat d'abord. Le titre est à l'union, le poème est à la fraction: le texte est le simultané battement, comme la feuille est le recto et le verso simultanés, du littoral et du littéral par l'encre et l'eau, celle-là plagiant celle-ci. Les flots sur la plage et la pénétrant, puis - mais, désormais, en même temps - les mots sur la page et la pénétrant aussi, la page qui est déjà littéralement dans la plage, qui est déjà littérale littoralement. La page blanche donne à la plage son sable blanc, le grain de sable (27) donne à la page son grain: les mots-flots battent le(s) grain(s) de la $p($ l)age, indiquant là le champ (41) de l'écriture et de la vision qu'il faut en avoir, débattant tout le texte. Ce battement, de gauche à droite, des flots sur la plage, des mots sur la page, est la direction même de la lecture qui l'institue et qu'il institue. Des versvagues.

Sur la plage où j'ancre, écriverain, j'encre sur la page que si l'eau ne devient l'o, l'eau n'est lue l'eau. L'eau de la mer désaltère, l'o du vers altère. Du même et de l'autre. Le livre, lieu d'absorption d'une encre, d'une écriture, est lieu d'une lecture absorbante: l'eau n'est lue l'eau sans les lèvres qui la boivent. Lus les livres, bus les vers.

Incipit impitoyable: en ces trois vers se commence en même temps que se commente le texte et la naissance de son écriture. C'est exactement où j'écris que les mots-flots viennent battre: c'est que les mots à rive, n'étant pas là avant, dans les flux de l'écriture même, avancent dans la lecture. L'eau ne peut être lue que dans les livres qui la boivent. Pour la boire, mettre sur le vers l'o des lèvres. Dans cette strophe, on est à l'extérieur sur la plage et à l'intérieur dans le langage. Ici, on est à l'intérieur dans une maison (41), avec le lit, un drap, la vitre, à l'intérieur dans le langage. Les mots-flots font écume dont, à défaut d'une déesse peut-être, il naîtra une enchanteresse. Des diamants de leur plus belle eau, ils font couronne: si reine (et non reine du foyer: nul embrasement) elle est, celle qui nage, elle est sirène. L'écume de pierres précieuses faisant o autour de l'îlot le plus désertique, le plus inculte, le plus blanc, l'îlot-désert: flots (écume blanche), îlot (sable blanc), lit (drap blanc). Cette écriture noire, écriture de nuit, à vers blancs, envers blanc, a son avers noir, en vers noirs, écriture du matin: mots, papier, vers. L'écriture précieuse faisant (p)o autour de l'ceil fermé (peau pierre) comme autour du corps fermé (drap peau) quand tout - bavardages, vents, stéréotypes, clartés, poncifs, froids, vulgarités: abruptures de la vie quotidienne - vient vite casser ses phrases infra, se phraser sur la vitre au matin (et non au mat tain: nul miroir), brides anonymes collectives. Au matin, au mitan du lit, sur le drap, sur le papier, les flots de la mer des mots émiettent sur la plage de la vitre, éclaboussent, versant abruptement le fonds de leurs vagues de fond.

Plage et page: papier sablé. Paupière et vitre: papier de verre. Miroir (cf. 11), papier buvard. Diamant (cf. 17), papier carbone. Sur le papier 
sablé s'écrit une banalité, une phrase usée (l'eau n'est plus l'eau sans les lèvres qui la boivent) dont le texte sera le lieu, lieu commun, étant le papier qui la boit: cette phrase, comme en un miroir, s'y lira alors à l'envers, blanche. Ici l'exemple de l'apologue:

Mais supposons que le comparant démesurément s'accroisse et s'organise. Alors le principe métaphorique transforme sa fonction expressive en rôle générateur de fiction. La littérature classique n'ignore pas cette hypertrophie du cry parant: c'est l'apologue. Le comparant du vers "La raison du plus fort est toujours la meilleure" est fait des vingt-sept derniers vers de le Loup et l'Agneau. Sans doute l'exaltation subsiste-t-elle: en son développement, le comparant a pour mission d'illustrer (de rendre illustre) la maxime comparée. Mais, inversement, on peut dire, du précepte, qu'il est un modèle sifuationnel pour la fiction comparante. L'apologue joue un rôle crucial, on le sait, dans le roman moderne, sous le nom de mise en abyme ${ }^{3}$.

Sur le papier de verre s'inscrivent, en s'y fracassant, une multitude de lancées de langage, de reflets de langage dont tout le texte sera le diamant, étant le papier qui le cristallise: ces reflets s'y reproduiront, comme avec un papier carbone, à l'endroit, noirs. De l'abrasion à l'embrasure (le coin le plus mince (45)), tous ces papiers sont les papiers d'identité de ce texte.

Cet œil et sa paupière qui est îlot (Presque-anagramme d'œil) dans le lit, ce lit et son lit d'eau qui est îlot (anagramme de lit o) dans la maison, la maison et sa vitre qui est îlot dans l'îlot, l'îlot et son sable qui est îlot dans la mer. Mise en abyme de l'œil, qui lit à l'intérieur dans le texte.

9 les mots-flots qui donnent aux ruisseaux

10 cette voix mi-ouatée qu'on leur connaît

11 voix miroitée

12 vois comme je te vois moi qui pourtant ferme les yeux

13 sur le plan fragile de tes cheveux

14 moi qui ferme les yeux sur tout

15 pour voir tout en equilibre

16 sur la pointe microscopique du cœur

17 pointe diamantée des dimanches hantés

18 dis à m'enchanter et jusqu'à m'en noyer

19 de ces longs rubans de mots-flots

20 que tu déroules le soir entre tes seins

21 comme si tout un fleuve rampait à tes pieds

22 comme si les feuilles n'avaient pour les bercer

23 d'autre vent que celui de tes cils de soie lactée

La paupière qui te couvre l'œil (l'iris) comme un drap te couvre le corps (l'éros), de la mer des mots aux mots de la mer ${ }^{4}$, en passant par les ruisseaux et un fleuve. Les mots-flots créent les ruisseaux: cette voix mi-ouatée leur naît, à mi-mots (fibre de vers), mi-flots (water ou ouater), cette voix couronnée (roi et, bientôt, roi de cœur), trouée (mitée), elle mire ouatée, étouffée, presque fermée. Première naissance: celle d'une voix douce. Cette voix fait rimer miroiriquement l'homme et la femme: voix/ vois/moi (l'homme) et voix/dis/te (la femme). L'homme est vu, est dit par la femme qu'il voit, qu'il dit. Mon écriture regarde les mots-flots, leur voix miroitée, reflet de leur écriture, et écoute les ruisseaux: si les yeux sont les miroirs de l'âme, les mots-flots sont la voix miroitée des ruisseaux, 
les mots-flots sont les miroirs, non plus de l'âme, mais de la maison, de la mariée (25).

Vois qu'homme je te vois, moi qui pour tant ferme les (cils noirs de mes) yeux, a les yeux bandés sur le plus fragile de tes aveux, la plus fragile phrase île à ton chevet, la plus fragile pousse de tes cheveux (blonds comme les blés du champ ou marins ${ }^{5}$ comme les joncs, les quenouilles de la plage) à tes chevilles: de l'îlot de sable blanc, sans vert, en passant par les pics neigeux (29), puis les dunes (32) de sable blanc, l'hiver brûlant, au champ, en passant par la plus fragile pousse, puis les feuilles, le vert se multiplie (42), le printemps: feuillaison, foliation. Le folio s'imprime, le vert te multiplie. Moi qui surtout ferme les yeux sur tout (par connivence, refusant de voir ce qui vient) pour tout voir, pour voir tout, le macroscopique, en équilibre sur le microscopique, la pointe diamantée du cceur des dimanches hantés: moi, faisant se reposer, se déposer le fracas des phrases toutes faites pour qu'il en reste un (bon) fond, une lie quotidienne: et qui est libre de ne pas utiliser cette lie pour en faire un l-ivre, de ne pas la fictionnaliser en une littérature à l'eau de rose, à l'eau d'une rose, d'un diamant taillé en facettes à l'avers (en $T$ ou, plutôt, en $\uparrow$ ) et plat à l'envers (en 1 ) ? Pointe mic-rose-copique, pointe de Rrose Sélavy, alter ego de Marcel Duchamp ${ }^{6}$, qui voudrait nous faire voir la vie en rose. Pointe aimantée, d'une boussole par exemple ${ }^{7}$, pour tenter de retrouver son chemin dans le jeu d'écarte: cœur (amant: roi de cœur), pique (amante: dame de pique), et atouts dans les manches ${ }^{8}$. Celui qui sait s'égarer sait se trouver un chemin, son chemin, dans l'écriture, son écriture.

Dimanches hantés vient de la fêlure de diamantée: plus ch, moins a. Dis à m'enchanter vient de la fêlure de dimanches hantés : plus a. Microscopiques. Dimanches hantés: dans le temps, hiatus charnière, jour d'équilibre obsédant entre deux fracassantes semaines; dans l'espace, poupe d'un navire fantôme qui donne de la bande d'un bord et de l'autre. Le vide et le plein. Le dit amant voit pour voir sur la pointe de la voix, l'écriture microphonique: une parole. L'équilibre, presque silencieux. La déc(h)antation. Quelque chose comme la sécheresse. La dite amante dit pour enchanter, ensorceler, enrubanner (comme il dit pour endimancher), subjuguer, submerger (cf. 41) par la longueur des flots de mots, l'écriture macroscopique. Quelque chose comme la fraîcheur, presque la fertilité. La luxuriance. Longs rus, longues bandes d'un magnétophone, longues bandelettes de mots mis à faire se dérouler la momie, précisément entre tes seins, longs rubans encreurs d'une machine à écrire: c'est que la mariée est une chevelure (flots de cheveux noués de flots de rubans), une secrétaire (à taire le secret: l'écume noire des flots de rubans encreurs imprimant le soir sur les feuilles l'écume blanche des mots), un antique cadavre desséché, une moderne enchanteresse: une voix, un ensemble de minces filets (rubans) d'eau ne se retenant plus de laisser s'écouler la trame d'un fleuve, le tissu de cette voix, par laquelle il s'agit de se perdre. Avant de submerger l'espace intérieur (table, lit) et extérieur (maison, champ), l'eau submerge l'espace interne du langage: noyer c'est d'abord noyer Noé, 
qui échappa à une autre noyade et, pour ce faire, être né o, né de l'o des mots.

Entre tes seins: tes seins bobines, ta tête liseuse du ruban qui bat près de la pointe du cceur, et l'affolement de la machine. Les rus deviennent un fleuve, les rubans deviennent un serpent, le fleuve-serpent rampe aux pieds de la vamp-Vierge: elle ne l'écrase pas, comme dans l'iconographie populaire, mais le laisse aller, le laisse produire, pour l'avoir produit. Cette machine, à la fois machine à enregistrer la parole et machine à écrire la voix, produit un foisonnement de feuilles. Les mots-fleuve (les pieds d'une moderne métrique), les feuilles-flots (l'apaisement, comme en un berceau de mots, le berceau de Moïse, d'une naissante écriture), feuilles dactylographiées, feuilles d'arbre, feuilles de température: température d'une lecture lactée, à base de lait, nourrissante, fertile. Blancs cils soyeux (et non plus autrement blanche paupière désertique, œil). Voir (cils) et boire (lactée) les mots-flots. Vent de sel, vent salin de la mer, vent lui (et non mat), miroité, des ciels de soirs lactés, ciels de nuits blanches, d'aurore boréale, de voie lactée. Lacs des yeux, lacis des mots-flots: entrelacs d'amour. Seconde naissance: celle d'une lecture douce.

24 les mots-flots toujours les mots-flots

25 sur le sable la mariée toute nue

26 attend la grande main salée de la marée

27 et un seul grain de sable déplacé démasque soudain

28 la montagne de la vie

29 avec ses pics neigeux ses arêtes lancinantes

30 ses monts inconquis ses cimes décimées

31 un seul grain de sable et ce sont aussitôt

32 des milliers de dunes qui apparaissent

33 puis des déserts sans mirages

34 un sphinx d'ébène

35 et trois cents pyramides humaines mortes de soif

36 un seul grain de sable et la mariée n'est plus à elle

37 ne s'appartient plus

38 devient mère et se couche en souriant

39 comme un verre renversé perd son eau

40 et les mots-flots envahissent la table

41 la maison le champ

42 le verre se multiplie par sa brisure

43 et le malheur devient transparent

44 semblable au matin qui entre

45 par le coin le plus mince d'un miroir sans tain.

Les mots-flots m'enflant, tout un fleuve mène à la mer. Sur la plage blanche, la mariée toute nue, mise à nu dit Marcel Duchamp, exposant le grain de sa peau, tend la main, attend que la grande main du sel y batte terre, la grande main de la marée, y batte sable ${ }^{9}$. Grande main ${ }^{10}$, marée haute. Main macroscopique, microscopique grain de sable. Grande main salée, presque-anagramme de seul grain de sable. Salée, presque-anagramme de déplacé. La grande cible de ce grain de sable est la mariée. Dans la mariée est littéralement la marée: la mariée possède le phallus, le i, 
une seule lettre fait déclic, dont le point, un seul grain de sable, déclenche trois simultanés instantanés. Trois gains de texte. Au bas mot, l'm hauts flots : la montagne (28), des milliers de dunes (32), la mariée (36). De quatre en quatre" Main de sel déplace un seul grain de sable, fait bague de sel. Mariage blanc. Main de scripteur déplace une seule lettre et coupe le vers, fait montage de coupes: coupe de neige et de pierre, coupe d'eau et de sable, coupe de champ(agne) et de terre. Mariage mousseux. En prise, clichés: cliché de la montagne de la vie, cliché de la vie au désert, cliché de la vie à la maison. La montagne de la vie: cette étendue verticale, toutes ces hauteurs (quatre: pics, arêtes, monts, cimes), s'accumuler en un $(1+1+1+1)$. La vie au désert: cette étendue horizontale, toute cette sécheresse (quatre: dunes, déserts, sphinx, pyramides), se succéder en un $(1,1,1,1)$. La vie à la maison: cette étendue globale, toute cette dépense (quatre: mère, verre, mots-flots, matin), produire et perdre un $(1 \rightarrow 1 \rightarrow$ $1 \rightarrow 1)$.

S'inverser le précédent mouvement: à la fois tout/en équilibre/pointe du cœur (cf. 15-16) et grain de sable/déplacé/montagne, et à tes pieds (21)/avec ses pics. II s'agit de fabriquer un nouveau verre avec ce sable, de nouveaux vers avec ce grain. Du sable au verre, du blanc au vert, le vers aura traversé la page blanche. Se chercher déjà un lecteur nouveau. De la vitre (8) où tout se phrase, la voix miroitée (11) où tout se jase, la pointe diamantée (17) où tout se tait, à la coupure: sous tain le verre, le montage de la vitre, soudain le vers, la montagne de la vie. S'écrire un cliché. Dénudation progressive, plus radicale: paupière drap (6), yeux fermés de l'amant (cf. 12-13), rubans entre tes seins (19-20), yeux ouverts de l'amante (cf. 23), mariée toute nue. La démasque, la fait apparaître en la désignant pour ce qu'elle est, une phrase montagne, en la mettant en état d'investir l'écriture: se faire une montagne de la vie, c'est se faire l'écriture d'un cliché, proposer un phrase toute faite qu'il s'agit de défaire et de refaire en la faisant travailler pour qu'elle produise son envers: une montagne qui accouche d'une souris (cf. 38). Telle souris n'est pas incompatible avec tel raz de marée: celui-ci, si violent et si infime (un seul grain de sable), et celle-là, si décevante (elle perd son eau) et si envahissante. Ici, propose de se prendre en mains propres la fiction, propose, comme en mains autres endroits, la fictionnalisation d'une phrase toute faite: celleci, produite, met en forme l'écriture de celle-là. II ne s'agit plus de représenter l'écriture (thème de l'écriture), mais de la faire (fictionnalisation de l'écriture). La faire, c'est se faire.

Cette grande main démasque soudain le dédain, la hauteur, l'amont de la vie. La vie, précisément, n'est que l'aval de l'amont, que l'écriture d'une écriture. Des pics sont aussi des oiseaux grimpeurs, nichant dans des trous d'arbre et se nourrissant surtout de vers. Des arêtes sont aussi des arêtes de poisson: avoir une arête dans la gorge, ce passage étroit entre deux arêtes ${ }^{12}$. Ici, les flots; là, les mots. Des monts sont aussi des merveilles: monts et merveilles, mont de Vénus, mont Everest ${ }^{13}$, mer. Des cimes sont aussi des cimes d'arbre: feuilles. Ici, les mots; là, les flots. 
De pics neigeux: pics à pointe aiguë et neige, et bics à pointe fine et encre blanche, à cimes décimées: cimes décimées au sens d'écimées, découronnées, décimées au sens de péries en grand nombre, et rimes dérimées, usées, non productrices, il est possible maintenant d'y lire un important renversement. Ce poncif, la montagne de la vie, produit d'exclusifs ponts qui, traversés, permettent l'écriture de son inversion: l'encre blanche de ses pics est celle, ici, du découronnement de ses cimes ${ }^{14}$. Des rimes nouvelles, productrices, mettent en écho ce poncif et l'amont du texte, pour en faire paraître l'aval: les mots-flots faisant couronne stérile au plus désertique îlot (cet îlot est un stérile o), par le déplacement d'un seul grain de sable se déclenche l'écriture de cette terre île qu'un style o bille habille, injecte, dévoile. L'amont: ses pics, n'ai-je (qu')eux, ses arrêts de lents silences, ses mots en coquille, ses rimes dérimées? L'aval : ce champ, cette multiplication, ce vers qui éclot de sa coquille, les rimes productrices de ce rimoir sans tain (cf. 45). Cette écriture, qui a les mains blanches, démasque une batterie d'armes blanches: massue (démasque), pique (pics), dard (arêtes), lance (lancinantes), cimeterre (cimes), faux (flots), sabre (sable). Cette écriture, qui a des conquêtes, touche le corps des femmes: tête, seins, raies, mont de Vénus. Jusqu'à l'adéquation entre l'objet de la conquête: cimes décimées.

Là, cimes décimées; ici, pyramides humaines mortes de soif - en fin de strophe. Les mots, les monts (montagne, monts, dunes, pyramides), la mort. Les flots, les flancs (lancinantes, humaines), la soif. Là, blanc comme sel, neigeux; ici, comme mort de sel, déshydraté. Ici, un sphinx d'ébène ${ }^{15}$, espèce de statue de sel noir, lion couché à tête d'homme, de bélier ou d'épervier; là, la mariée se couche (38). Ici, ce son, aussitôt: des milliers de dunes liées à partir d'une, par réaction de chaîne en chaîne de montagne. Dunes continentales, à l'intérieur des déserts, s'opposant justement aux dunes maritimes, au bord des mers. Dunes continentes, appâts à paresse. Puits sans eau des déserts sans faux miroitements de nappes d'eau (cf. 40). Puits des désirs inassouvis, des images sans rimes, des $r$ sans mirages: ici, mi-ouatée ne devient plus miroitée. Ce sable fin se fixe en un bois noir, dur et uni, couché sur l'étendue. Le blanc ne le boit pas. II incarne le roi pharaon ou le dieu Soleil. Telle absence d'ébène, noire, dure comme le diamant, d'une présence multitude humaine, blanche: nul, un sphinx étroit sans trois cents pyramides (presque-anagramme de puis mirages), taillées comme des diamants, où sont inhumées, monuments tombeaux des pharaons, les sèches lèvres esclaves qui les ont érigées. Grain du bois, grain de la pierre, grain de la peau: pertes sèches. Si l'eau n'est plus l'eau sans les lèvres qui la boivent, les pyramides humaines ne sont plus des pyramides humaines si elles ne sont pas mortes de soif. Là, il faut être bu; ici, il faut boire. Là, l'eau salée sur la plage, mais l'eau douce sur la page des livres et les lèvres de la page. Ici, l'eau douce bue par les esclaves, mais l'eau salée bue par les déserts, les pyramides étant de continentales mers mortes ${ }^{16}$. Ce renversement, $s^{\prime} i l$ désigne le travail de l'écriture qui dévoile ses inversions, se retourne aussi lui-même en en désignant un autre: le vers renversé. 
L'amante et l'amant (cf. 17-18), la mariée et la marée (25-26), la mère et le père. Un seul grain de sable dans l'engrenage où nage l'encre, et la machine d'écriture n'est plus à elle: le vers est renversé. Un seul spermatozoïde, grain de sable de ce père mat aux eaux salées, dans sa vulve, son vagin, et la mariée est l'avariée, n'est plus à elle, est la mal liée, ne se tient plus et ne tient plus sa part, mais appartient déjà à ce vers qui se multiplie. Ça part, elle se secoue, ce coup, en s'ouvrant, ce coût, perd son o, se brise, s'éparpille comme le verre, les lèvres. Se dépense, se perd. Perd la tête (ct. 30) comme un verre perd son eau. Elle a son i, le père son 0 : la marée sort de son lit et vient la coucher, et vient l'accoucher, elle se laisse aller à la renverse dans son lit, devient mère et verre (mère/qu'homme/verre), vers et mer (vers/comme/mer). Recouvre tout. Verre eau, motsflots, vers mer: elle génère des mots-flots comme les mots-flots produisent cette fiction en et par laquelle elle est générée. Elle en a l'eau à la bouche, le sourire aux lères.

La grande main a pris un verre, un grand verre est renversé: le Grand Verre (1915-1923), retard en verre de sept ans et quelques mois en quelque sorte, couché dans un camion de Brooklyn à Redding en 1927, multiplié en cours de route, constaté en 1931, réparé en 1936, en quelque sorte retard en verre de neuf ans et quelques mois, de Marcel Duchamp.

Un verre est renversé. II perd son eau (39-41), il se brise (42-43). La multiplication de l'o (mots-flots) et la multiplication du vers (dissémination), simultanées. De grains de sable, c'est à grains de verre qu'il se produit en et par la brisure de sa fabrication, cellules vivantes proliférant vers, à la lettre. Aussi, u seul, verre vers ${ }^{17}$ tous, y lire: se couche en souriant comme un verre renversé perd son eau. Couche en: $u, n$. Son eau: $n, u$. Double inversion: d'une part, se couche, eau serait renverser leur $u$ en $n$, et en, son serait renverser leur $\mathbf{n}$ en $u$; d'autre part, c'est à la lettre qu'ici les extrémités se referment. Souriant, un: un, un. Annulation. Renversé: $n$. Un verre, $u$, est renversé, $n$. Le verre dans lequel l'eau n'est plus l'eau sans les lèvres qui la boivent perd son eau: ce n'est plus la page et son papier qui la boit, mais l'écriture et son texte. La mer se brise sur les brisants, la mère se brise dans le fonctionnement de ses fonctions, se multiplie dans ce qui n'est pas elle: l'écriture. Ni pertes blanches, ni pertes sèches. L'écriture, à la lettre de cette lettre et de cette phrase toute faite, commence. Giguerriers, les mots-flots envahissent la table de la fable, la maison de mes sons, le champ du chant. lls font nappe d'eau (cf. 33) sur la table de nuit: ils servent à boire et à voir le malheur.

Un verre est renversé. Un miroir est brisé ${ }^{18}$ : sept ans de malheur ${ }^{19}$, comme les dimanches, septièmes jours, de hantise (17), comme trois cents pyramides pires. Plus le verre se brise, plus le malheur.devient transparent: plus le ver travaille, plus le lien d'opaque causalité, d'irrépressible représentation est travaillé, est fictionnalisé. Plus le malheur, les maux sont transparents, plus le vers, les mots sont écrits: plus l'écriture se et le(s) prend en charge, coupant et occupant tout le territoire, toute l'audition. A la superstition d'un poncif, croyance populaire, répond précisément l'exorcisme 
d'une écriture, travail populaire. Au déluge répond le nouveau monde. Le matin du nouveau jour, par degrés, entre et se multiplie comme le verre, par le coin le plus mince d'une vitre transparente comme le malheur. Là, tout vient se fracasser sur la vitre, comme s'arrêter sur elle. Ici, le matin entre par la vitre: sa traversée, dont le malheur devient trans-parent, est de table de nuit à écriture de jour, envahissement travaillé de l'intérieur par l'extérieur ${ }^{20}$.

Comme le Grand Verre, transparent intransportable, ce miroir sans tain, ce tiroir sans main, ce rimoir s'entend non comme la reproduction d'une transcendance, d'une totalité d'emblée donnée, mais bien comme la réduction au plus mince de cet au-delà du sens (grand malheur, grande noirceur), la fabrication d'une écriture nouvelle, écriture à la lettre, rimoir au coin le plus mince ${ }^{21}$. Vers multipliés, dans l'angle grandissant du livre, blancs, semblables au sable blanc d'une $p($ (I)age nouvelle, lue écrite par les mots-flots du matin, où regarde déjà l'écoute moderne. Vers d'eau, de toute façon.

\section{La paille du texte}

Le texte à la paille, la paille du texte: quelques notes sèches sur quelques lieux comme un

L'importance de la phrase toute faite, proverbe, adage, de l'expression toute faite, poncif, cliché, n'est pas nouvelle chez Giguère. Dès le début, il travaille, inégalement, ces (ses) couches de langage. II s'agit ici de travailler à faire s'écrire: de passer de l'expression (décoration/défoulement) stylistique à la production textuelle, le texte s'écrivant de se lire, se formant de se transformer. Au dessus et en dessous. Le texte a des surfaces textuelles: au dessus et en dessous. Au dessus a des miroitements de signifiants par en dessous. Au dessus organise, en l'élaboration de son tracé, les travaux d'en dessous. L'un n'est pas le signifié de l'autre.

"L'Homme à la paille 22 "

1 II vécut vingt ans avec une paille dans l'œil

2 puis un jour il se coucha

3 et devint un vaste champ de blé

Ce court texte exemplaire propose d'emblée un élément reconnaissable, telle locution proverbiale venue de la Bible: il voit la paille dans l'œil du voisin et ne voit pas la poutre dans le sien. Mais avoir une paille dans l'œil, c'est avoir dans l'œil un corps étranger. Et devenir un champ de blé, c'est devenir un corps étranger. S'établit alors une équivalence:

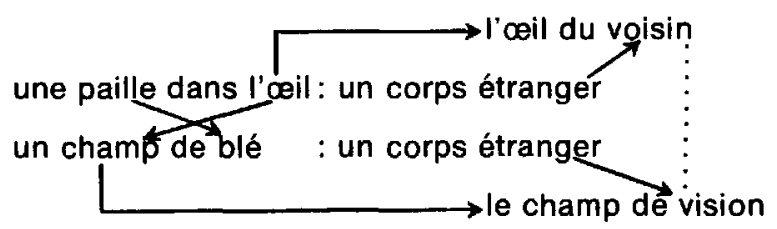


L'œil et le champ, l'œil du voisin et le champ de vision, par contiguïté. L'œil devient le champ par la paille qui devient blé: œil pour champ, paille pour blé. Mais la paille, dit-on, est lettre morte et, de plus, n'est pas le grain. Se propose alors ${ }^{23}$ telle autre locution proverbiale venue de Leibniz: séparer la paille des mots et le grain des choses. Et c'est précisément avec la paille des mots, avec l'écriture, que l'œil devient le champ. Mais l'un ne se couche pas sans l'autre. Aussi se propose tel poncif venu d'Éluard: les yeux fertiles. L'œil fertile, à faire s'écrire la fertilité dévastante de son travail, produit littéralement le champ de blé.

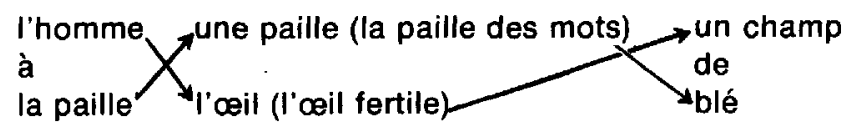

Une paille dans l'œil, un homme dans la terre, du blé dans le champ.

Texte en abyme, texte en expansion. Production du texte.

$$
\begin{aligned}
& \text { paille } \rightarrow \text { homme: blé } \\
& \text { œil } \rightarrow \text { «terre»: champ }
\end{aligned}
$$

Ce blé de l'œil, en aval, un autre texte au moins, "les Yeux du pain ${ }^{24}$ ", en propose d'intéressantes alluvions:

comment manger le pain

quand le couteau perce les mille yeux de la mie?

[...]

rien que les blés couleur d'un regard

sur un corps sans blessure.

L'œil fertile de l'homme à la paille est, ici, les mille yeux de la mie du pain. Fertile/mille, I'homme/la mie, la paille/le pain.

$$
\begin{aligned}
& \text { "blé" } \rightarrow \text { blés: mie } \\
& \text { corps } \rightarrow \text { regard: yeux }
\end{aligned}
$$

Le blé de l'œil, d'une écriture de l'œil, est, ici, la lecture des blés, le regard des blés sur un corps censure, corps sans blé (corps assemblé, accord semblé), corps sans blessure. Si l'œil n'est pas blessé mortellement à l'écriture, le regard n'est pas blessant, ne se forme pas de transformer le corps à la (sa) lecture. Nul aveuglement, mais les mille yeux d'une écriture blessée, en la mie du milieu du corps de l'amie. Pain ${ }^{25}$, pénétration. Plus avant dans le texte. Coupure:

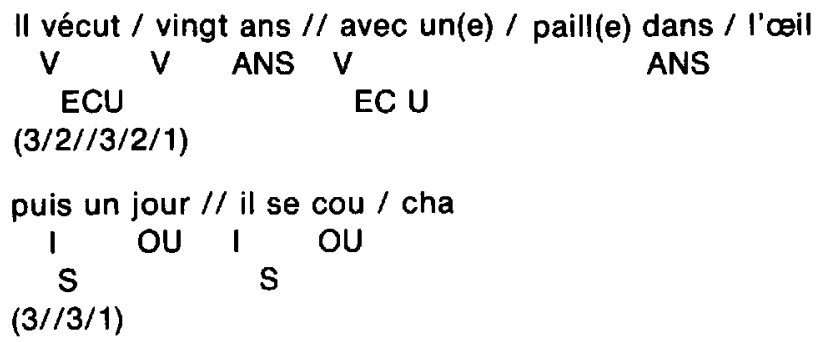


et / devint / (t)un vas / te champ / de blé.

DE IN T TE AN DE

$(1 / 2 / 2 / 2 / 2)$

$$
V \quad V \quad B
$$

Autre:

II vécut dâns l'œil

se coucha

et devint de blé.

Autre:

l'œil

ce coup

de bléén.

Un homme servit de prête-œil à une paille dans cette affaire. II ne fut pas un homme de paille, il ne fut pas empaillé, il ne se coucha pas sur la paille, mais il vécut vingt ans ${ }^{27}$, morsure de la paille, et devint un vaste champ, mort sûre de l'ceil, le v devenu un b: le blé. Ecceil.

D'autre part, si "La main du bourreau finit toujours par pourrir ${ }^{28}$ ", c'est que le défaut de la main du bourreau est d'avoir des panaris. Et si Le défaut des ruines est d'avoir des habitants ${ }^{29}$, c'est que l'édifice finit toujours par tomber en ruines. La main pourrir comme la paille, nous lever (le v) comme le blé. Aller ailleurs. Les poutres de l'édifice pourrir, l'édifice s'écrouler. Habiter les ruines d'ici. Cette tension, puisqu'il l'écrit. Ailleurs, cet ici; ici, c'est ailleurs.

André Gervais

\section{Le troupeau des mots : essai de traduction-réécriture du poème ales Mots-flots" par Antoine Di Nardo ${ }^{30}$}

II s'agit d'offrir un équivalent du titre:

\section{LES M/OTS-FL/OTS.}

$\begin{array}{llll}\downarrow & \downarrow & \downarrow & \downarrow \\ \mathrm{a} & \mathrm{x} & \mathrm{b} & \mathrm{x}\end{array}$

Tout de suite, la traduction littérale:

THE W/ORD-W/AVES.

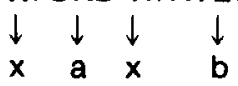

(Remarquons ici qu'en français les deux noms communs sont d'égale force: les mots sont des flots et les flots sont des mots ${ }^{31}$, tandis qu'en anglais le premier terme devient un adjectif et ne prend donc pas d's: les mots sont des flots.) Mais il faut trouver un autre mot que WAVES pour retrouver le dispositif et la disposition du son (ax-bx). S'offre alors

THE W/ORD-H/ERDS (Les mots-troupeaux).

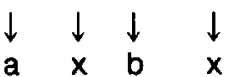


Cette équivalence sonore et presque graphique entraîne un changement tel qu'un nouveau texte doit être écrit ${ }^{32}$. Particulièrement, telle série, liée à FLOTS, devient, liée à HERDS:

\section{FLOTS}

plage (vers 1)

eau (vers 2)

îlot (vers 4)

ruisseaux (vers 9)

noyer (vers 18)

fleuve (vers 21)

main salée de la marée (vers 26)

soif (vers 35)

eau (vers 39)
HERDS

plain (plaine)

herbs (herbes)

pond (mare)

hills (collines)

smother (étouffer)

valley (vallée)

green hand of the prairie (main verte de

la prairie)

green thirst (soif verte)

sound (son)

Ajoutons aussi que, de la même façon que le son $\mathrm{O}$ est commun à MOTS et FLOTS et que l'eau ( $\mathrm{O}=\mathrm{eau}$ ) est l'un des ressorts principaux du texte de départ, le son ERD est commun à WORD et HERDS et le son (ERD = heard, ce qui est entendu) est l'un des ressorts principaux du texte d'arrivée, page suivante.

\title{
Remarques sur la traduction
}

vers 1 : battre la plage blanche/trample the pale plain-page les $B, P, L$ du texte français sont repris dans les $P, L$ du texte anglais; la plage(page) devient the plain-page (la plaine-page); plain page, sans trait d'union, est un équivalent de page blanche (blank sheet en serait un autre): ici, pour blanche est ajouté pale (cf. vers 4,25 ).

vers 2: herbs est à plain ce que eau est à plage; herbs, herds, words, comme eau, flots, mots.

vers 4: pond est dans plain exactement comme, mais inversé, îlot est dans eau;

unflowered indique qu'il n'y a jamais eu de fleurs, renforce

deserted, rime avec word, et s'inscrit dans la série des un-:

unless (vers 3 )

unwind (vers 20)

unmasks (vers 27)

unconquered (vers 30)

unsilvered (vers 45)

\author{
ct. aussi \\ on (vers $13,14,16,25)$ \\ hundred (vers 35)
}

vers 5: bed-pond, d'où swim.

vers 6 : sheet, à la fois feuille (de papier) et drap (cf. vers 22).

vers 7: dans versant il y a vers, comme dans incline il y a ink line (d'où inkline).

vers 8 : vitre/pane of glass

pane rappelle plain (vers 1 ) et peut aussi se lire pain (cf. vers 43 ); glass, crystalline glass, unsilvered mirror, comme vitre, verre, miroir sans tain: tout ça est trans-pa-rent (cf. vers 43). 


\section{«LES MOTS-FLOTS}

Les mots-flots viennent battre la plage blanche où j'écris que l'eau n'est plus l'eau sans les lèvres qui la boivent

les mots-flots couronnant le plus désertique îlot

5 le lit où je te vois nager la nuit et la paupière qui te couvre comme un drap au versant abrupt du matin quand tout vient se fracasser sur la vitre

les mots-flots qui donnent aux ruisseaux

10 cette voix mi-ouatée qu'on leur connaît voix miroitée

vois comme je te vois moi qui pourtant ferme les yeux sur le plus fragile de tes cheveux moi qui ferme les yeux sur tout

15 pour voir tout en équilibre sur la pointe microscopique du cœur pointe diamantée des dimanches hantés dis à m'enchanter et jusqu'à m'en noyer de ces longs rubans de mots-flots

20 que tu déroules le soir entre tes seins comme si tout un fleuve rampait à tes pieds comme si les feuilles n'avaient pour les bercer d'autre vent que celui de tes cils de soie lactée

les mots-flots toujours les mots-flots

25 sur le sable la mariée toute nue attend la grande main salée de la marée et un seul grain de sable déplacé démasque soudain la montagne de la vie

30 ses monts inconquis ses cimes décimées

un seul grain de sable et ce sont aussitôt des milliers de dunes qui apparaissent puis des déserts sans mirages un sphinx d'ébène

35 et trois cents pyramides humaines mortes de soif un seul grain de sable et la mariée n'est plus à elle ne s'appartient plus devient mère et se couche en souriant comme un verre renversé perd son eau

40 et les mots-flots envahissent la table la maison le champ le verre se multiplie par sa brisure et le malheur devient transparent semblable au matin qui entre

45 par le coin le plus mince d'un miroir sans tain. 


\section{«THE WORD-HERDS "}

The word-herds come trample the pale plain-page where I write that herbs are no longer herbs unless lifted to the lips

the word-herds crowning the pale pond deserted and unflowered

5 the bed where I see you swim at night and the eyelid that covers you like a sheet at the abrupt inkline of morning when everything comes crashing at the pane of glass

the word-herds which give the hills

10 this sound halt-hounded that we know them from sound resounded see how I see you I who nonetheless close the eyes on the most fragile of your hairs I who close the eyes on everything

15 to see everything in equilibrium on the microscopic point of the heart diamond-pointed with mined ointments meant to enchant me and as far as smothering me in these long ribbons of word-herds

20 that you unwind in the evening between your breasts as if a valley slithering at your feet as if the leaves but had to lull them another wind other than that of your eyelashes of milken silk

the word-herds always the word-herds

25 on the pale sand the naked bride awaits the great green hand of the prairie and a single grain of sand displaced unmasks suddenly the moutain of life with its snow caps its racking ridges

30 its unconquered peaks its decapitated pinnacles

a single grain of sand and as soon thousands of sand dunes appear then deserts without mirages an ebony sphinx

35 and three hundred human pyramids dead of green thirst a single grain of sand and the bride is no longer hers does no longer belong to herself becomes mother and goes to bed smiling like a crystalline glass overturned loses its sound

40 and the word-herds invade the table the house the field the crystal line multiplies itself through its shattering and the misfortune becomes transparent like the morning entering

45 by the thinnest corner of an unsilvered mirror. 
vers 9: hills est à herds ce que ruisseaux est à flots.

vers 10: voix mi-ouatée/sound half-hounded

en français l'accent est mis sur la douceur, en anglais sur la frayeur; ici, deux sens: une voix qui vous poursuit comme si elle vous (pour)chassait, une voix qui hurle et qui presque vous hante.

vers 11: voix miroitée/sound resounded

sound s'entend et se "voit» dans resounded comme dans un miroir: resounded est à l'oreille ce que reflected est à l'œil.

vers 17: mined ointments (onguents-parfums tirés de la terre), leur aspect exotique, ésotérique même; mined s'ajoute aux multiples références topographiques (plain, hills, etc.) et peut se lire aussi mind, s'opposant ainsi à heart (vers 16); plus les sonorités jouent, plus les mots, d'une langue à l'autre, peuvent s'éloigner: ici, l'accent est mis, comme dans le texte de départ, sur la reprise serrée des sons, mais surtout sur le lien entre les surfaces (plain, hills, etc.) et les profondeurs (mined), et le «conflit créateur» entre mind et heart.

vers 18: smothering (étouffant, comme la poussière derrière les troupeaux en marche peut étouffer le lecteur), à rapprocher de slithering (vers 21), another et other (vers 23), becomes mother et smiling (vers 38).

vers 21 : valley est aussi between your breasts.

vers 22: leaves, à la fois feuilles (des arbres) et feuilles de papier (cf. vers 1, 6).

vers 25: naked est toute nue, nude serait nue;

sand, ici, est l'absence de verdure (cf. vers 4).

vers 26 : les $A, E, R, M, L$ du texte français sont repris dans les $R, E A(A I) E E(I)$ du texte anglais; green indique et permet le passage de plain (vers 1) à pond (vers 4) puis à sand (vers 25); green s'oppose à pale, comme la fécondité s'oppose à la stérilité.

vers 27 : dans single il y a sing (cf. vers 10);

grain s'ajoute à great green, et sand à hand.

vers 29-30: ici, les S,C(K),P,R;

cimes décimées devient decapitated caps.

vers 31-32: ici, les S,N,D,A,AN,OON.

vers 39: crystalline met l'accent sur la pureté, la richesse (cf. diamond, vers 17), la transparence (cf. vers 43) et la fragilité du son;

crystalline glass overturned, à la fois verre «cristallin" renversé et son cristallin dont le volume est trop haut (d'où shattering, vers 42).

vers 41 : field s'ajoute très bien à la série plain, hills, valley, prairie, etc.

vers 42: dans le texte de départ, ce vers, comme par exemple le vers 1, est très important: il se lit aussi

le vers se multiplie par sa brisure

d'où la nécessité, dans le texte d'arrivée, d'indiquer les deux lectures: dans crystalline (vers 39) il y a crystal line comme dans incline il y a ink line (vers 7), comme dans plage (vers 1) il y a page. 
vers 39-45: accumulation de préfixes, dans tous les sens: overturned, invade, transparent (trans- est repris dans through), entering, unsilvered.

vers 43: the misfortune peut se lire the miss for tune (le manque [qui est pris] pour la mélodie): cela met définitivement l'accent sur le son (cf. sound, vers 10) et, surtout peut-être, sur le vide, le désert (cf. deserted, vers 4), le manque (miss) qui entre par là, c'est-àdire par le son, dans tout le texte et l'emplit dans tous les sens, comme le matin entre par la vitre, ce miroir sans tain, et emplit tout le jour.

vers 43-45: accumulation de OR,AR,ER,IR.

1. "La Seine" (écrit en 1947, publié en 1950), in Tome premier, Paris, Gallimard, 1965, p. 525.

2. "Pour aller plus loin: ne jamais demander son chemin à qui ne sait pas s'égarer" "Grimoire", écrit en 1955, publié en 1957, in Le défaut des ruines est d'avoir des habitants, Montréal, Éditions Erta, 1957, p. 74). Plus loin : demander. Avis: déranger. Acquis: s'égarer. Ici, Stéphane Mallarmé: «ll peut avancer, parce qu'il va dans le mystère" («igitur", écrit en 1867-1870, publié en 1925, in (Euvres complètes, Paris, Gallimard, "Bibliothèque de la Pléiade ", 1945, p. 450). Le mystère dans les lettres, à la lettre. Faut-il ajouter que les quelques "Notes sur la poésie" (écrites en 1963, publiées en 1964, in Guy Robert, Littérature du Québec. Poésie actuelle, Montréal, Déom, 1970) sont d'un idéalisme constant: on y parle de poète éclaireur, de poète profond, de poète habité, de poète "Voleur de feu », de poème inspiré, de poésie mystérieuse. J'appelle idéaliste ce léger effort théorique (appels-citations de Rimbaud, Breton) qui est constamment freiné par l'effort passionnel d'être surréaliste et qui ne se laisse pas d'être inarticulé à - donc dépassé par - sa pratique en ce qu'elle offre et retire d'être (re)lue.

3. Jean Ricardou, Pour une theorie du nouveau roman, Paris, Seuil, «Tel Quel», . 1971, p. 119.

4. Qui ne deviennent pas les maux de la mère (cf. 38) et le malheur de la vi(tr)e, la brisure du vers et les flots des mots s'écrivant de la brisure du verre et des reflets du miroir. L'écriture sait, au matin, qui être et quoi faire, quoi être et qui faire.

5. Cheveux marins, et aussi chevaux marins (hippocampes): miroitée se dit aussi d'un cheval à la robe brun rouge, des taches plus brillantes qu'il a à la croupe.

6. De la rose, diamant (ou la rrose : cet $r$ qui donne à cette voix mi-ouatée sa tessiture miroitée), au champ, vert, un certain travail d'écriture, un certain travail de jardinage est nécessaire: jardinage du diamant, fêlure et taches, défaut; jardinage du champ, sillons, culture et entretien, défi.

7. Cf. "sans autre boussole que la pointe vibrante du cceur, constamment en éveil "( «Yeux fixes", écrit en 1950, publié en 1951, in l'Âge de la parole, p. 94).

8. Cf. «je pique en plein cœur/et voici le lit où naîtra le printemps" («Jeu d'eau du rêve", écrit en 1952, publié en 1965, in l'Age de la parole, p. 22). Un renversement.

9. Cf. "Sur le rivage la marée vient soulevant ses seins/et s'en retourne avec ses vagues déchiquetées/dentelle d'eau salée sur nos poitrines meurtries” (" Jeu d'eau du rêven $n$ ). Un renversement.

10. Cette grande main, faut-il le dire, n'est pas celle, bien connue, de «La main du bourreau finit toujours par pourrir" (ecrit en 1951, publié en 1960, in l'Âge de la parole, p. 17). Main de la marée n'est pas main du bourreau. Grande main n'est pas grand demain (I' "ailleurs", dernier mot de ce texte, pourrait être oppose, en ce sens, à l'«ici», dernier mot de «Recours didactique», 
in la Vie agonique (1963) de Gaston Miron). Main transformatrice n'est pas main inhibitrice. Main introduisant tel jeu n'est pas main d'emblée là, que travaillera telle inflammation aiguë. Un renversement: là, "les jointures éclateront comme des verres de cristal»; ici, «le verre se multiplie par sa brisure" (42).

11. Notre division du texte: 8 vers, 15 vers, 22 vers. Plus sept, plus sept. Ici, mais nulle cabale.

12. D'arêtes à crêtes: des crêtes sont aussi des crêtes de vagues. Ici aussi, les flots.

13. Le mont Everest est conquis le 29 mai 1953. Ici, des monts d'écriture inconquis (30), il reste toujours - ever rest.

14. De pics à cimes, de $\mathrm{c}(\mathrm{k})$ à $\mathrm{c}(\mathrm{s})$, de $n$ à $\mathrm{m}$ :
pics $n$ e i ge ux
a rêtes la n c i na ntes
monts incon quis
c i $m$ es d éc $c$ i m é es
i, seules pointes qui n'aient pas été émoussées, à la lettre.

15. Cf. les «sept grands pharaons d'ébène" du "Tombeau des rois", in le Tombeau des rois (1953) d'Anne Hébert. L'Égypte: sphinx, pyramides, directement; désertique, sable, dunes, déserts, indirectement; papier (cf. 6), qui vient du grec papuros "roseau d'Égypte, ce jeu de cartes appelé pharaon (ct. 16-17), momie (cf. 19), plus indirectement encore. Ici, telle petite égyptologie textuelle.

16. Cf. "Certains soirs de grand désert quand le désespoir erre entre les dunes, il arrive d'entendre un chant mystérieux comme un appel de sirène en mer morte" ("Visages vécusn, écrit en 1956, publié en 1958, in la Main au feu (poèmes 1949-1968), Montréal, Éditions de l'Hexagone", « Rétrospectives ", 1973, p. 88).

17. De verre et de vers, une valise où se jouent les langues dans la poignée peut faire verse qui en français renvoie au premier et en anglais au second. Ici, mais le texte en est ren-verse-é.

18. Cf. "nos consciences sont éparpillées dans les débris/de nos miroirs, nos gestes des simulacres de libertés" (Recours didactiques"). Recours didactique, dit tactique d'écriture à frôler, par le coin le plus mince, le non-poème.

19. Cf. "pour avoir une image claire de l'homme/tous les ans il fallait briser sept miroirs " ("l'Effort humain ", onzième poème des Armes blanches, in l'Âge de la parole, p. 121). Un renversement.

20. Cf. "Les dunes s'évanouissent et l'horizon apparaît bien assis sur la paupière; on ouvre les yeux, on ouvre la nuit, et le matin entre par la porte de sortie: (Lettres à l'évadé, écrit en 1951, publié en 1957, in la Main au feu, p. 42). Et aussi : "Pour entrer dans l'intimité des choses: se faire infiniment petit " ("Grimoire", in Le défaut des ruines est d'avoir des habitants, p. 72). Des renversements.

21. D'un seul grain de sable déplacé (27), ça part, à la lettre, dans une circulation où se (re)prend tout le texte jusqu'ici: et un Seul gRain de SAble déPLACé démASque Soudain (27). Avec Ses Pics neigeux Ses ARêtes lancinantes (29). APPARaiSSent (32), et thOls Cents PyRAmides (35), un Seul gRain de SAble et IA mARiée n'est Plus A elle, ne S'APPARtient Plus (36-37), un veRRe RenveRSé PeRd Son (39), Se multiplie PAR SA (42), tRanSPARent (43), PAR le coin le plus minCe (45).

22. Troisième poème des Nuits abat-jour (10 poèmes écrits en 1949-1950, publiés en 1950), in l'Âge de la parole, p. 67.

23. Si le texte met en scène une fiction, le dictionnaire est, avec la grammaire et la rhétorique, par exemple, un fictionnaire, lieu indéfini de répliques, d'outils, de langage(s).

24. Neuvième poème des Armes blanches, in l'Âge de la parole, p. 117.

25. En français il renvoie à la mie et en anglais à la blessure. Fleur de farine et défloration.

26. C'est, en clin ou en coin : le blé

$$
\text { ce coup }
$$

$$
\text { d'œil. }
$$

27. En 1949, Giguère a vingt ans.

28. Titre d'un poème (1951).

29. Titre d'un ensemble (1957), avant l'Âge de la parole (1965) et la Main au feu (1973).

30. Antoine Di Nardo pour la traduction et une première rédaction de l'introduction et des notes; André Gervais pour une seconde rédaction de l'introduc- 
tion et des notes, relue par Antoine Di Nardo; Thunder Bay, Ontario, juillet 1974.

31. Dans le texte français, l'accent est mis directement sur FLOTS et $\approx$ indirectement " sur MOTS. Par exemple, dans les vers 1-3:

Les mots-flots viennent battre la plage blanche

où j'écris que l'eau, n'est plus l'eau

sans les lèvres qui la boivent

plage $\rightarrow$ page; plus $\rightarrow$ lue, lèvres $\rightarrow$ livres

d'ou, aussi et en même temps,

Les mots-flots viennent battre la page blanche

où j'ecris que l'eau n'est lue l'eau

sans les livres qui la boivent.

LES MOTS-FLOTS c'est, à la fois, les jeux de mots-le jeu des flots et le jeu des mots-les jeux de flots.

32. Texte qui a été élaboré tout à fait en dehors des traductions déjà existantes: "The Word-Waves»; version anglaise de Jean Beaupré et Gael Turnbull, dans Eight Poems by Roland Giguere, Iroquois Falls, Ontario, 1955; "Words Waves", traduit par George Ross Roy, dans Twelve Modern French Canadian Poets, Toronto, Ryerson Press, 1958; "The Wave-Words" traduit par John Glassco (publié une première fois en 1965, dans The Poetry of French Canada in Trans/ation, publié par John Glassco, Toronto, Oxford University Press, 1970, auxquelles nous renvoyons le lecteur. 